\title{
L'ALIMENTATION DES CARPILLONS D'UN ÉT'É PENDANT L'HIVER
}

\author{
Par M. W.-K. TARAN \\ Chef de la Section dihydrobiologie, \\ à l'Institut scientifique ukrainien de Recherches pisctcoles.
}

Plusieurs auteurs faisant autorité dans la littérature piscicole assurent que, dans la règle, la Carpe ne s'alimente pas durant la saison froide. Daprès WaLten et Ar.vold, elle cesserait de rechercher sa nourriture lorsque la température s'abaisse au-dessous de $6^{\circ}-8^{\circ} \mathrm{C}$. Les jeunes sujets aussi seraient soumis à ce jeûne et passeraient l'hiver engourdis sur le fond.

D'après Susta, cette suspension de l'appétit serait la conséquence de certaines modifications saisonnières affectanl l'épithélium intestinal qui deviendrait, Iransiloirement, non fonctionnel (I).

Quoiqu'il en soit au juste, en raison de celle parlicularilé biologicque qui leur est attribuée, les carpillons nés durant l'élé sont, en général, placés à l'automne dans de petits viviers spécialement aménagés où on se préoccupe sculement d'assurer le renouvellement de l'cau. La conviction que, comme les adultes, jls mènent, en état d'hibernation, une vie ralentie, incite à ne prendre aucun souci de leur alimenlation.

Cependant, depuis quelques années, les exploitants des étangs de l'Ukraine ont élé amenés, par l'observation, à concevoir des doutes sur la réalité de cette anorexie des carpillons.

Pour savoir à quoi s'en tenir, des investigations furent entreprises par lit Section d'hydrobiologic de l'Instilut scicntifique de Recherches piscicoles, dont le siège est à Kiew. Elles ont eu licu dans les bassins d'hivernage do deux élevages voisins de celte ville, à lworetz et à Ssowski, d'abord en Mars-Avril rg $3_{1}$, ensuite de Janvier à Arril r932.

Ic nombre des sujets pèchés, alors que la lempéralure de l'cau était comprise entre $3^{\circ}$ et $8^{\circ} \mathrm{C}$. s'est élevé à r 48 .

Sur ce nombre, 34 présentaient, dans leur intestin, des aliments d'origine animale plus ou moins altérés par la digrestion, nolamment des Copépodes (Cyclops, Canthocamplus) et des larves de Diptères (Chironomus). Chez un individu, furent trouvés, presqu intacts, Irois vers de terre (Lumbricus).

Pour -3 autres carpillons, les traces furent relevées d'une nourriture a base végćtale : débris de plantes aquatiques et grumeaux informes de vase.

(1) La phénomène serait donc à rapprocher de celui de l'anorexie génétique. Voir, à ce sujet : - Bulletin, $\mathrm{n}^{\circ} 3 \overline{7}$, Juillet $\mathrm{r}^{3} \mathrm{3}$, p. 5 
Enfin les voirs digrestives de 41 poissous étiient vides à l'autopsie, comple non teru d'un mucus abondant.

Plus tard, quand la tempéralure de l'eau s'éleva à $7^{\circ}$ - $1^{\circ}$ C. (25 Avril

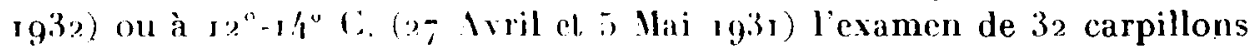
révéla la présence, en sus des Enlomostracés et larves d'Insectes plus haut signalés, de nombreux vers (Nématodes ou Oligochètes) ainsi que de feuilles d'une Mousse (Fontinalis).

Les constatiations failes permellent done d'affirmer qu'au cours de leur premier hiver les jeunes Carpes sont à même de s'alimenter quand la température est in féricure à $6^{\circ}-8^{\circ} \mathrm{C}$. el encore quand elle s'abaisse à $3^{\circ} \mathrm{C}$. ou moins. Du reste, le fait a élé déjà signalé par P. Scumemenz dès i 925.

Non seulement les aliments sont absorbés, mais ils sont assimilés. La preuve en est fournic par leur aspect, lors des autopsies. Dans la partic antérieure de l'inlestin ils apparaissent intacts ou fort peu altérés ; dans lit partic postéricure subsistcul sculs, encore reconnaissables, des débris chitineux, provenant de la carapace pour les Copépodes, de l'armature céphalique pour les Vers de vase. Fin se référant aux travaux de Tammax, Schönmor el Wikтomow, Bickre, Poskrski, Ilinzor et autres, sur l'activite optimale de divers enz̧̧mes aux basses températures, il est très explicable que les carpillons soicnt en siluation d'utiliser la nourriture ingérée en hiver. En oulre, il faut tenir compte du rôle essentiel que jouent, daprès Zuntz el Cиомнеiм, les enzymes fournies par les orçanismes qui constituent les proies. Cependant il liaut reconnaitre qu'en temps de froidure, les phénomènes de la digestion sont fortement ralentis et que l'assimilation est incomplète.

D'après ce qui vient d'être crposé, l'absence dans les viviers d'hivernage des animalcules susceptibles d'être consommés par les jeunes Carpes ne peut manquer d'exercer sur clles une influence nuisible, étant donné qu'en gónéral elles y séjouruent cinq à six mois. Ce n’est pas le seul inconvénient, car il n'est pas rare que les poissons aient à pâtir d'autre manière, notam. ment en raison de la faible teneur de l'eau en gaz et sels dissous. L'insuffisance d'oxycène ou d'éléments minéraux provoque le rachitisme ou l'ané. mie, et favorise l'apparition et le diveloppement de diverses maladies souvent meurtrières (saprolégniase, cyclochaetiase, chilodoniase, costiase, etc...). Ainsi, dans quelques piscicultures russes, a-t-on enregistré de r 930 à r $93 \%$, des pertes moyennes de 60 à $80 \%$ des effectifs et même, dans plusieurs cas, de po à ron \%. Pour obvier à un déchet d'empoissonnage aussi énorme, il importe de prendre des mesures.

Il ne paraît plus admissible que les carpillons d'un été soient maintenus au régime jusqu'ici adopté pour l'hivernage, car il implique une diselte raractérisée des ressources alimentaires que peuvent fournir la faune ou la More aquatiques. I notre avis, il conviendrait d'héberger ces poisons, durant la mauvaise saison, dans des étangs d'élevage proprement dits, 
de I mètre à $I^{m}$ jo de prolondeur, asséchés et cultivés pendant l'été. Leur remplissage s'effectuerait seulement un mois el demi à deux mois avant la mise en charge; de la sorte s'y multiplieraient les organismes animaux ef végélaux dont les élèves se repaitraient au cours de l'hiver.

Celte méthode aurait encore l'avantage d'améliorer l'état chimique du milieu où ils vivent, comme aussi l'état sanitaire.

On peut escompter, par conséquent, que son adoption permettrait d'éviter les pertes anormalement élevées qui, avec le système actuel des viviers d'hivernage surpeuplés, éprouvent fréquemment les carpiculture dans les régions comme l'Ukraine.

\section{BIBLIOGRAPHIE}

Walter (E.). - Karpfennutzung in kleinen Teichem. - Neudamm, 1928. Walter (E.). - Richtlinien für Karpfenfütterung. - Neudamm, 1928.

Anvold. - Osnovi prudovogo chosjaistva. - Moscou, I93r.

ELconsk. - Rybowodswo ; Razvedenie Karpov v prudach. - Moscou. 1925 .

Susta (J.). - Die Enährung des Karpfens und seiner Teichgenossen. Stetlin, I905.

Srhismexz (F.). - Die Ausnutzung des Süsswassers durch die Fischerei. Handbuch der biologischen Arbeitsmetode. - Schwarzenberg, i 925. Tammax. - Zeitschrift für physilkalische Chemie, HI. - Leipzig, $188_{9}$.

Tamman. - Hoppe Seylers Zeitschrift für physiologische Chemie. - XVI. - Strasbourg, i 892 .

Tamman. - Rousskogo Chimitscheskogo Obschetswa. - XXIV. - SaintPétersbourg, 1892 .

SchöndonF \& Wintonow : - l'fü̈gers Archiv für die gesamte Physiologie des Menschen und der Tiere. CXVI. - Bonn, 1907.

Pozenskr. - Comptes rendus hebdomadaires des séances el Mémoires de la Société de Biologie, L.II. - Paris, rgoo.

Bıскег. - Deutsche medizinische Wochenschrift. IXXII. - Leipzig, I9ö. Hinzog. - Hoppe Seylers Zeitschrift für physiologische Chemie. XXXVII. - Strasbourg, rgo3.

Zuxtz \& Cronuen : - Die Bedeutung der Naturnährung für die Ernährung der Teichfische. - Deustche Fischerei. - Veudamm, ig I .

DETAIL DES ILTOPSIES DE I 48 CARPILLONS DE LNN FTE F. PROVENANCE DES PISCICULTI RES DE DWORETZ FT SSOWKI EN UKRAJNE

$$
\text { I. - Pisciculfure de Dworetz. - Bassin } n^{\circ} 3 \text {. }
$$

A. - 3o Mars r 93 г. - Inventaire du contenu intestinal de ro Carpillous.

Grumeaux neu abondants de vase d'un noir-verditre (4). - Débris rle plantes aqualiques (3). - Imas de mucus (1). - Particules chitinenses de Coléopteres (cuisses, 
inneaux de l'abdomen) (1). - Cyclops intact du groupe de C. serrulatus (I). - Hésidus chitineux de Cyclops sp. ? (2). - Résidus chitineux de Canthocamplus sp.? (2). Cuticule et lébris céphaliques chitineux de larves de Chironomides (3). - Quelques traces d'Algnes filamenteuses (Cladophora) et de biatomacces.

La cavité intestinale de 6 poissons était entièrement vide, mais ses parois revêtues d'une couche de mucus.

B. - 4 Avril 1931 . - Température de l'eau : $2^{\circ}, 6-3^{\circ}, 6$ l: - Inventaire du contenu intestinal de 10 Carpillons de 4 i à 108 grammes.

Grumeaux peu abondants de vase (3). - Détri!us peu abondauls de plantes aquaLiques (4). - Cyclops Dybowstii intact (1). - Résidus chitineux de Cyclops macruroides (3). - Résidus chitineux de Canthocamptus sp.? (1). - Résidus chitineux d un Insecte de la famille des Haliplidés (r). - Quelques algues : Euglena sp). ?, (yymbella maculata.

$$
\text { II. - Pisciculture de Ssurosly. - Bassin n०9. }
$$

C. - 25 Mars I $_{9}$ r. - Inventaire du contenu intestinal de so carpillons de $1 \mathrm{I}-63,5 \mathrm{gr}$.

Grumeaux peu abondants de vase (5). - Résidus chitineux de circlops bicuspidalus, C. macrunotdes et $C$. strenuus var. abyssorum avec œufs intacts de Cyclops sp. ? (I). - Cuticule de ver de vase (1).

La cavité intestinale de 3 poissons ditit absoluntent vide

D. - i6 Avril i $9^{31}$. - Température de l'eau : $0^{\circ}, \$-3^{\circ}$ C. - Inventaire du contenu intestinal de wo carpillons de so, $\overline{3}-26, \overline{\mathrm{gr}} \mathrm{gr}$

Grumeaux de vase (4). - Poils isolés (4) ou en peloton! (I) ... Détritus peu abon. dants de plantes aquatiques (1). - Filaments mycéliens isolés de Saprolegnia (3). 3 verrs de terre (Lumbricus) intacts ( 1 ). - Résidus chitineux de 54 individus de Cyclops sp. ? (ri) - 4 exemplaires à demi digérés de lyclops bicuspadatus et auland de C. sp. ? (1). - Résidus chitineux de Cyclops sp. ? (r). - Késịdus chitineux de Coléoplère aqualique (cuisses, élytres) (i). - Ctuls intacte de Cyclops sp. ? (1),

E. - 27 Avril 1935 - Température de l'ean : $12^{\circ}$ C. - Insentaire du contenu intestinal de a carpillons.

Grumoaux peu abondants de vase (j). -- Particules de coquilles de Mollusques (2). - Débris de la peau d'Oligochètes (2). - Vers de vase (2). - Quelques Nématodes intacts (4). - Cyclops biruspidatus intacł ou à demi digcéré (1) - Quelques résidus chitineux de Cyclops sp ? (2). - Résidus chitineux de Canthacamptus sp. ?. Feuilles et tigelles de Mousse (Fontinalis) isniées el rares.

F. - 4 Mai r93ı. - Température de l'eau : $15^{\circ}, 7-16^{\circ}$, , C. - Inventaire

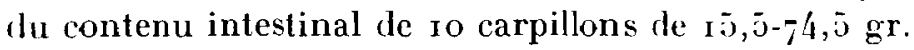

Grumeaux de vase peu abondants (1). -- upelques libres végélales et débris de plante (r). - Rares résidus chilineux de rycclops bicuspidatus (h). -- Résidus chilineux de Cyclops sp. ? ahondants (1) et rares (1). - Résidus chitineux isolés de Canthocamptus sp. ? (2). - Quelques résidus chitineux d'Insecte (1). - Tématodes isolé: el intacls (2). - Débris de la peau d'oligochìles (1). - Cuticule et résidus chitineus de Chironomides (4). - Feuilles et tigelles de Mousse (Famlimilisi. - Carapace de Bosmina longirostris (2). - Lne carapace de Brachionus urcells el de B. caliciflorus amphiceros (I). - Rares résidus chilineux de Colćoptère (i). -- Plantes aqualiques isolées, mais abondantes (Nilzschia, Synedra, Navicula, OXjogonius, Gomphonrla. Olivaceus, Cymbella, Cocconeis, Trachelomonas, Meridion, Irinnularin, Olivacens, Cosmarius, etc.). 


$$
\begin{gathered}
-2 \mathrm{Q1}- \\
\text { III. - I'isciculture de Sswosly. - Bassin } n^{\circ} 10 .
\end{gathered}
$$

(;. - itj Ivril rg3r. - Tempéralure de leau : $3^{\circ}$ C. - Inventaire du contenu intestinal de 2 carpillons de 19,5 et $24,5 \mathrm{gr}$.

Grumeaux de vase (1). - Débris d'organismes végétaux (2). - Joils (1). - Filaments myoéliens de Saprolegnio (2). - Résidus chitineux de 6 exomplaires de Cyclops bicuspidatus (r). - Orufs intacts de Cyclops sp. ? (I).

II - 27 Avril r931. - Température de l'eau : ı $2^{\circ}$ C. - Inventaire du contenu intestinal de ro carpillons de $25,5-108,5 \mathrm{gr}$.

Grumeaux de vase contenant des particules de copuilles de Mollusques (1). - Rares fibres végétales (I). - Nématodes et Oligochètes intacts et à demi digérés, abon. dants (8). - Quelques débris de la pean d'Oligochètes $(7)$. - Quelques cuticules et résidus céphaliques chitineux de vers de vase (5). - Quelques exemplaires intacls le Cyclops bicuspidalas et C. sp. ? (3). -.. Résidus chitineux le Cyclops bicuspidatus el C. sp. ? (2). - Résidus chilineux de Canlhocamplus sp.? (1). - Feuilles de Mousse (Fonlinalis) (4).

$$
\text { II. - Pisciculture de Dworetz. - Bassin } n^{\circ} 12 .
$$

I. - 9 Janvier r $93 \%$ - Inventaire du contenu intestinal de 7 carpillons de $\mathbf{~}, 6-3,3$ gr.

Grumeaux de vase (5). - Détritus végétaux (4). - Hares fibres végétales (3). Rares Diatomacées (6). - Algues aquatiques (1). - Une carapace de Chydorus (3). Résidus chitineux de Canthocamptus (r).

J. - 28 et 29 Février r $93 \%$. Inventaire du contenu intestinal de ro carpillons de $2-6,7 \mathrm{gr}$.

Grumeaux de vase (3). - Fibres végétales nombrcuses (5). - Quelques débris végé taux (5). - Cladophora abondante ou rare (3). - Quelques carapaces de Chydorus (2). - Résidus chitineux de Canlhocamptus ( $\mathrm{I}$ ).

K. - i I-I . Mars $193 \%$ - Inventaire du contenu intestinal de so carpillons de $3,7-7 \mathrm{gr}$.

Gruineaux de vase (7). - Quelques fibres végétales (8). - Quelques carapaces de Chydorus (3). - Cyclops à demi-digéré ( $\mathrm{r}$ ). - Résidus chitineux de Cyclops sp. ? ( $\mathrm{I}$ ). - Cuticule de Asplanchna sp. ? (1). - Carapace de Brachionus ( $\mathrm{I}$ ). - Résidus chitineux d'Insectes ( $\mathrm{r}$ ). - Débris abondants de Melosira ( $\mathrm{r}$ ). - Diatomacées en abon. dance $(\mathrm{r})$.

$$
\text { V. - Pisciculture de Ssowsky. - Bassin } n^{\circ} 5 .
$$

L. - 3 Février 1932. - Inventaire du contenu intestinal de I carpillons de $6,9-3 \mathbf{r}, 9 \mathrm{gr}$.

Grumeaux de vase (2). - Plantes aquatiques abondantes (I). - Fibres végétales (II). - Détritus de plantes (I).

Y. - 27 Février 1932 . - Invenlaire du contenu intestinal de so rarpillons de $9,3-40, \mathrm{r}$ gr.

Grumeaux de vase (1)..- Fibres végétales (9).... l)étritus de plantes abondants ( 1 ) et rares (8). - Cladophora fragments presque intacts ou peu altérés et très nombreuses tnveloppes de cellules ( $\mathrm{I}$ ). 
Y. - i Mars ig3s. - Inventaire du contenu intestinal de r.I carpillons de $7,8-34,8 \mathrm{gr}$.

Grumeaux de vase peu abondants (1i). -- Quelques détrilus végélaux (1 i) .- Statoblaste de Plumatella ( $\mathrm{r}$ ).

O. - 8 Avril rg $32 .-$ Température de l'eau : $3^{\circ}-4^{\circ} \mathrm{C}$. Inventaire du contenu intestinal de 20 carpillons de 2,3-71,9 gr.

Grumeaux de vase ${ }^{\prime}$ ). - Détritus végétan: (1). -- Fibres végétales (1). - Résidus chitineux de Cyclops sp. ? (1). - Carapaces de Chylorus sp. ? (1). - Résidus chiti. neux céphaliques de vers de vase (s). - Statoblasto de Plumatelle (1).

$$
\text { VI. - Piscicullure de Ssousliy. - Bassin } n^{\circ} 6 \text {. }
$$

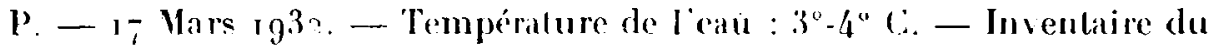
contenu intestinal de 11 carpillons de $\mathrm{s}, 5-3,9 \mathrm{gr}$.

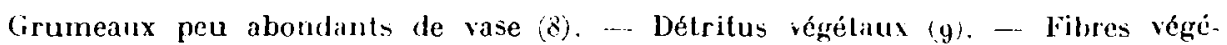
tales (8). - Cuticules et résidus céphaliques chitineux de vers de vase (1) - Spirogyra (1). - Rares Diatomacées ( $\mathrm{r}$ ).

(2. - 8 Avril rg3a. - Température de l'eau : $3^{\circ}-4^{\circ}$ C. - Inventaire du contenu intestinal de wo carpillons fr $\$, 8-98,9 \mathrm{gr}$.

Grumeaux de vase (1). - Fibres végétales isolées (b). - Détritus végétanx (2). -. Résidus chitineux d'Insectes (2). - Cyclops a divers degrés de digeslion (6). - Carapaces de Cladocìres (6). - Résidus chitinenx de Coléoplìres (r). - Díbris de larves de Chironomides (Chironomus, Culicoides, Tanypus: (8) - Oufs intacts de Cyclops sp. ? (1). - Nématores ( 1 ).

B. - ע. Svril rg3?. - Température de l'eau : $7^{\circ}-1^{\circ}$ C. -- Inventaire du contenu intestinal de 7 carpillons de $2-3 \mathrm{gr}$.

Grumeaux de vase, détritus végélaux et fibres végébaliss en plus ou moins granrle abondance (-). - Cyclops intacts (3). - Bcsmina longirostris, B. cornuta et Chydorus sp.? exemplaires un peu altérés (n). - Résidus chitineux de vers de vase (3). - . ligues aquatiques (3). - Volvox (r).

- Erratum. - L'ne erreur a été commise dans le compte rendu de l'Exposition de Poissons vivants d'eau douce au Salon international d'Iviculture de rg36, pour la rescription des trois bacs de l'Association des Producteur; de Carpes d'élite ( $\mathrm{I}$ ).

Il faut lire :

8. - Carpe ì miroir marque "Royale ". - Sujets de consommation de 2 livres issus de feuilles de 5 grammes analogues à celles, nées en ra 35 , exhibées avec eux.

9. - Carpe à miroir marque "Royale ". - Sujets de consommation de 3 livres issus de feuilles de 3o grammes analogues a celles, nces en 1935 , exhibées avec eux.

10. - Carpe à miroir marque "Royale ". - Sujets de consommation de 5 livres issus de feuilles de 50 grammes analogues à colles, nóes en 1933 . exhibées avec eux.

( $\mathrm{r})$ Voir Bulletin : $-n^{\circ} 9^{3}$. Mars $\mathrm{r} 936, p . \mathrm{r} 8 \mathrm{f}_{\text {. }}$ 